VOL. $71(2005)$ [505-516]

\title{
THE GROUP OF POISSON AUTOMORPHISMS OF POISSON SYMPLECTIC SPACE
}

\author{
Sei-Qwon Oh and Eun-Hee Cho
}

The group of Poisson automorphisms of the coordinate ring of Poisson symplectic $2 n$-space is isomorphic to the algebraic torus $\left(\mathbf{k}^{*}\right)^{n+1}$ and it confirms that the algebra constructed by K.L. Horton (2003) is a quantisation of the coordinate ring of Poisson symplectic $2 n$-space.

\section{INTRODUCTION}

In [6], Horton constructed a class of algebras $K_{n, \Gamma}^{P, Q}$ which includes the multiparameter quantised coordinate rings of symplectic and Euclidean $2 n$-spaces, the graded quantised Weyl algebra, the quantised Heisenberg space, and is similar to a class of iterated skew polynomial rings constructed by Gómez-Torrecillas and Kaoutit in [4]. As a Poisson case, in [9], the first author constructed a class of Poisson algebras $A_{n, \Gamma}^{P, Q}$ which includes the coordinate rings of Poisson symplectic and Euclidean $2 n$-spaces and whose quantisation is $K_{n, \Gamma^{\prime}}^{P^{\prime}, Q^{\prime}}$ for suitable $\Gamma^{\prime}, P^{\prime}$ and $Q^{\prime}$. Moreover Gómez-Torrecillas and Kaoutit proved in [5] that the group of automorphisms of the coordinate ring of quantum symplectic $2 n$ space is isomorphic to the algebraic torus $\left(\mathbf{k}^{*}\right)^{n+1}$. The main purpose of this paper is to find the group $H$ of Poisson automorphisms of the coordinate ring of Poisson symplectic $2 n$-space which is isomorphic to the algebraic torus $\left(\mathbf{k}^{*}\right)^{n+1}$. This confirms that $K_{n, \Gamma}^{P, Q}$ is a quantisation of $A_{n, \Gamma}^{P, Q}$.

This paper consists of three sections. In the first section, we review several elementary but basic and important properties which are used in the next sections. In the second section, we define $H$-actions on $A_{n, \Gamma}^{P, Q}$ which act as Poisson automorphisms and prove that every $H$-prime Poisson ideal is generated by an admissible set. In the final section, we prove that the group of Poisson automorphisms of the coordinate ring of Poisson symplectic $2 n$-space is just $H$ defined in the second section.

Received 10th March, 2005

The first author is supported by the Korea Research Foundation Grant, KRF-2002-015-CP0010.

Copyright Clearance Centre, Inc. Serial-fee code: 0004-9727/05 \$A2.00+0.00. 


\section{The Poisson algebra $A_{n, \Gamma}^{P, Q}$}

1.1 Let $\Gamma=\left(\gamma_{i j}\right)$ be a skew-symmetric $n \times n$-matrix with entries in $\mathbf{k}$, that is, $\gamma_{i j}=-\gamma_{j i}$ for all $i, j=1, \ldots, n$. Let $P=\left(p_{1}, p_{2}, \ldots, p_{n}\right)$ and $Q=\left(q_{1}, q_{2}, \ldots, q_{n}\right)$ be elements of $\mathrm{k}^{n}$ such that $p_{i} \neq q_{i}$ for each $i=1, \ldots, n$. Then, by $[9,1.2]$, the polynomial ring $\mathbf{k}\left[y_{1}, x_{1}, \ldots, y_{n}, x_{n}\right]$ has the following Poisson bracket:

$$
\begin{aligned}
\left\{y_{i}, y_{j}\right\} & =\gamma_{i j} y_{i} y_{j} & & (\text { all } i, j) \\
\left\{x_{i}, y_{j}\right\} & =\left(p_{j}-\gamma_{i j}\right) y_{j} x_{i} & & (i<j) \\
\left\{y_{i}, x_{j}\right\} & =-\left(q_{i}+\gamma_{i j}\right) y_{i} x_{j} & & (i<j) \\
\left\{x_{i}, x_{j}\right\} & =\left(q_{i}-p_{j}+\gamma_{i j}\right) x_{i} x_{j} & & (i<j) \\
\left\{x_{i}, y_{i}\right\} & =q_{i} y_{i} x_{i}+\sum_{k=1}^{i-1}\left(q_{k}-p_{k}\right) y_{k} x_{k} & & (\text { all } i)
\end{aligned}
$$

The Poisson algebra $\mathbf{k}\left[y_{1}, x_{1}, \ldots, y_{n}, x_{n}\right]$ is denoted by $A_{n, \Gamma}^{P, Q}$ or by $A_{n}$ unless any confusion arises.

One should observe that the class of multi-parameter algebra $K_{n, \Gamma^{\prime}}^{P^{\prime}, Q^{\prime}}$ constructed by Horton in [6] is a quantisation of the Poisson algebra $A_{n, \Gamma}^{P, Q}$, where $P^{\prime}, Q^{\prime}$ and $\Gamma^{\prime}$ are multiplicative forms for $P, Q$ and $\Gamma$, respevtively.

1.2. In $A_{n}$, set

$$
\Omega_{i}=\sum_{k=1}^{i}\left(q_{k}-p_{k}\right) y_{k} x_{k}
$$

for each $i=1, \ldots, n$ and let $\mathcal{P}_{n}=\left\{\Omega_{1}, y_{1}, x_{1}, \ldots, \Omega_{n}, y_{n}, x_{n}\right\} \subseteq A_{n}$. A subset $T$ of $\mathcal{P}_{n}$ is said to be admissible if it satisfies the following conditions:

$$
\begin{aligned}
& \text { 1. } y_{i} \text { or } x_{i} \in T \Leftrightarrow \Omega_{i} \text { and } \Omega_{i-1} \in T \quad(2 \leqslant i \leqslant n) \\
& \text { 2. } y_{1} \text { or } x_{1} \in T \Leftrightarrow \Omega_{1} \in T .
\end{aligned}
$$

1.3. An element $a$ of a Poisson algebra $A$ is said to be normal if $\{a, A\} \subseteq a A$. Note that $a A$ is a Poisson ideal if $a$ is normal.

LEMMA. In $A_{n}$, we have the following:

$$
\begin{aligned}
& \left\{y_{i}, \Omega_{j}\right\}=-q_{i} y_{i} \Omega_{j}, \quad\left\{x_{i}, \Omega_{j}\right\}=q_{i} x_{i} \Omega_{j}, \quad(i \leqslant j) \\
& \left\{y_{i}, \Omega_{j}\right\}=-p_{i} y_{i} \Omega_{j}, \quad\left\{x_{i}, \Omega_{j}\right\}=p_{i} x_{i} \Omega_{j}, \quad(i>j) \\
& \left\{\Omega_{i}, \Omega_{j}\right\}=0 \text {, } \\
& \text { (all } i, j \text { ) } \\
& \Omega_{i-1}=\left\{x_{i}, y_{i}\right\}-q_{i} y_{i} x_{i}, \quad \Omega_{i}=\left\{x_{i}, y_{i}\right\}-p_{i} y_{i} x_{i}
\end{aligned}
$$

Hence, all $\Omega_{i}, i \geqslant 1$, are normal elements of $A_{n}$ and $y_{i}$ and $x_{i}$ are normal modulo the ideals $\left\langle\Omega_{i}\right\rangle$ and $\left\langle\Omega_{i-1}\right\rangle$.

Proof: See $[9,1.3]$. 


\section{LEMMA 1.4 .}

(a) For every admissible set $T$, the ideal $\langle T\rangle$ is a prime Poisson ideal of $A_{n}$.

(b) For every prime Poisson ideal $P$ of $A_{n}, P \cap \mathcal{P}_{n}$ is an admissible set.

ProOF: See [9, 1.5 and 1.6].

1.5. We define an order on the generators of $A_{n}$ by

$$
y_{1}<x_{1}<y_{2}<x_{2}<\cdots<y_{n}<x_{n}
$$

and give the grade lexicographic order on the set of all standard monomials

$$
y_{1}^{r_{1}} x_{1}^{r_{2}} y_{2}^{r_{3}} x_{2}^{r_{4}} \ldots y_{n}^{r_{2 n-1}} x_{n}^{r_{2 n}}
$$

of $A_{n}$, where $r_{i}$ are nonnegative integers. That is,

$$
\begin{aligned}
y_{1}^{r_{1}} x_{1}^{r_{2}} y_{2}^{r_{3}} x_{2}^{r_{4}} \cdots y_{n}^{r_{2 n-1}} x_{n}^{r_{2 n}} & \prec y_{1}^{s_{1}} x_{1}^{s_{2}} y_{2}^{s_{3}} x_{2}^{s_{4}} \cdots y_{n}^{s_{2 n-1}} x_{n}^{s_{2 n}} \\
& \Longleftrightarrow \sum_{1 \leqslant i \leqslant 2 n} r_{i} \\
\sum_{1 \leqslant i \leqslant 2 n} r_{i}=\sum_{1 \leqslant i \leqslant 2 n} s_{i}, r_{2 n} s_{i} \text { or } & =s_{2 n}, \ldots, r_{i+1}=s_{i+1} \text { and } r_{i}<s_{i} .
\end{aligned}
$$

Note that, for standard monomials $X^{\alpha}$ and $X^{\beta}$ of $A_{n}$ with $X^{\alpha} \prec X^{\beta}$, we have $X^{\alpha} X^{\gamma}$ $\prec X^{\beta} X^{\gamma}$ for all standard monomials $X^{\gamma}$, that is, the grade lexicographic order $\prec$ is a monomial order by [2, Section 2.2].

Fix an admissible set $T$ of $A_{n}$. For each $i=1, \ldots, n$, set

$$
\begin{aligned}
S_{i} & =\left\{j \mid 1 \leqslant j<i, \Omega_{j} \in T\right\} \\
i_{0} & =\max S_{i} \quad \text { if } S_{i} \neq \phi .
\end{aligned}
$$

For each $i=1,2, \ldots, n$, define an element $\Omega_{i}^{\prime} \in A_{n}$ by

$$
\Omega_{i}^{\prime}= \begin{cases}\Omega_{i}-\Omega_{i_{0}} & \text { if } S_{i} \neq \phi \\ \Omega_{i} & \text { if } S_{i}=\phi\end{cases}
$$

and denote

$$
G_{T}=\left\{x_{i} \mid x_{i} \in T\right\} \cup\left\{y_{i} \mid y_{i} \in T\right\} \cup\left\{\Omega_{i}^{\prime} \mid \Omega_{i} \in T, x_{i} \notin T, y_{i} \notin T\right\}
$$

Note that the leading terms of $\Omega_{i}^{\prime}$ and $\Omega_{i}$ are equal and if $\Omega_{i}^{\prime} \in G_{T}, i>1$, then $\Omega_{i-1} \notin T$.

In order to find a k-basis for $A_{n} /\langle T\rangle$, we use an argument for the Gröbner basis. Refer to [2, Chapter 2] for further background and terminologies on the Gröbner basis. 


\section{LEMMA .}

(a) For every admissible set $T$ of $A_{n}, G_{T}$ is a Gröbner basis for $\langle T\rangle$.

(b) The algebra $A_{n} /\langle T\rangle$ has a k-basis $\mathcal{B}_{T}$ consisting of the natural images of all the standard monomials which are not divided by any leading terms of elements in $G_{T}$.

(c) The elements $\bar{\Omega}_{j} \in A_{n} /\langle T\rangle, \Omega_{j} \notin T$, are algebraically independent over $\mathbf{k}$.

Proof: (a) Note that $\langle T\rangle=\left\langle G_{T}\right\rangle$ by the definition of admissible set. We use the notation given in [2, Chapter 2]. Arrange the elements of $G_{T}$ by the order induced by the following arrangement

$$
x_{n}, y_{n}, x_{n-1}, y_{n-1}, \ldots, x_{1}, y_{1}, \Omega_{n}^{\prime}, \Omega_{n-1}^{\prime}, \ldots, \Omega_{1}^{\prime} .
$$

Now the $S$-polynomials of the forms $S\left(x_{i}, x_{j}\right), S\left(x_{i}, y_{j}\right)$ and $S\left(y_{i}, y_{j}\right)$ are all zero and the $S$-polynomials of the forms $S\left(x_{i}, \Omega_{j}^{\prime}\right)$ and $S\left(y_{i}, \Omega_{j}^{\prime}\right)$ are clearly reduced to zero modulo $G_{T}$ by the division algorithm. Finally consider the $S$-polynomial of the form

$$
\begin{aligned}
S\left(\Omega_{i}^{\prime}, \Omega_{j}^{\prime}\right) & =\left(q_{j}-p_{j}\right) y_{j} x_{j} \Omega_{i}^{\prime}-\left(q_{i}-p_{i}\right) y_{i} x_{i} \Omega_{j}^{\prime} \\
& =\left(q_{j}-p_{j}\right) y_{j} x_{j} \Omega_{i-1}^{\prime}-\left(q_{i}-p_{i}\right) y_{i} x_{i} \Omega_{j-1}^{\prime} . \quad(i<j)
\end{aligned}
$$

Since $\Omega_{j}^{\prime}$ is the first element in $G_{T}$ such that its leading term divides each term appearing in $\left(q_{j}-p_{j}\right) y_{j} x_{j} \Omega_{i-1}^{\prime}$ and $\Omega_{i}^{\prime}$ is the first element in $G_{T}$ such that its leading term divides each term appearing in $\left(q_{i}-p_{i}\right) y_{i} x_{i} \Omega_{j-1}^{\prime}$, we have that

$$
\begin{aligned}
S\left(\Omega_{i}^{\prime}, \Omega_{j}^{\prime}\right) & =\left(q_{j}-p_{j}\right) y_{j} x_{j} \Omega_{i-1}^{\prime}-\left(q_{i}-p_{i}\right) y_{i} x_{i} \Omega_{j-1}^{\prime} \\
& =\left(\Omega_{j}^{\prime}-\Omega_{j-1}^{\prime}\right) \Omega_{i-1}^{\prime}-\left(\Omega_{i}^{\prime}-\Omega_{i-1}^{\prime}\right) \Omega_{j-1}^{\prime} \\
& =\Omega_{j}^{\prime} \Omega_{i-1}^{\prime}-\Omega_{i}^{\prime} \Omega_{j-1}^{\prime} .
\end{aligned}
$$

It follows that $S\left(\Omega_{i}^{\prime}, \Omega_{j}^{\prime}\right)$ is reduced to zero modulo $G_{T}$ by the division algorithm.

(b) It follows immediately by (a) and [2, Section 2.6 Proposition 1$]$.

(c) Let $\left\{\Omega_{i_{1}}, \ldots, \Omega_{i_{k}}\right\}=\left\{\Omega_{i} \mid \Omega_{i} \notin T\right\}$ and suppose that $0 \neq f \in \mathbf{k}\left[z_{1}, \ldots, z_{k}\right]$ such that $f\left(\bar{\Omega}_{i_{1}}, \ldots, \bar{\Omega}_{i_{k}}\right)=0$, where $z_{1}, \ldots, z_{k}$ are indeterminates. Let $\alpha$ be the coefficient of the leading term of $f$ under the grade lexicographic order of monomials for $z_{1}<z_{2}$ $<\cdots<z_{k}$. Since the coefficient of the leading term of $f\left(\Omega_{i_{1}}, \ldots, \Omega_{i_{k}}\right)$ under the grade lexicographic order of standard monomials given in 1.5 is equal to $\alpha \beta$ for some nonzero $\beta \in \mathbf{k}$ and $f\left(\Omega_{i_{1}}, \ldots, \Omega_{i_{k}}\right)$ is reduced to zero modulo $\langle T\rangle$ by the division algorithm via $G_{T}$, we have $\alpha=0$, which is a contradiction. Hence $\bar{\Omega}_{j} \in A_{n} /\langle T\rangle, \Omega_{j} \notin T$, are algebraically independent over $\mathbf{k}$.

\section{2. $H$-ACTIONS ON $A_{n, \Gamma}^{P, Q}$}

2.1. Denote

$$
H=\left\{\left(h_{1}, \ldots, h_{2 n}\right) \in\left(\mathbf{k}^{\times}\right)^{2 n} \mid h_{2 i-1} h_{2 i}=h_{2 j-1} h_{2 j} \text { for all } i, j\right\} .
$$


The multiplicative subgroup $H$ of $\left(\mathbf{k}^{\times}\right)^{2 n}$ acts on $A_{n}$ as follows. For $h=\left(h_{1}, h_{2}, \ldots, h_{2 n}\right)$ $\in H$ and $f \in A_{n}$,

$$
h \cdot f=f\left(h_{1} y_{1}, h_{2} x_{1}, \ldots, h_{2 n-1} y_{n}, h_{2 n} x_{n}\right) .
$$

Note that each element of $H$ acts on $A_{n}$ by a Poisson automorphism and $H$ is isomorphic to the algebraic torus $\left(\mathbf{k}^{*}\right)^{n+1}$.

2.2. Let $A$ be a Poisson algebra and let a group $G$ act on $A$ by Poisson automorphisms. A proper Poisson ideal $Q$ of $A$ is said to be $G$-prime Poisson ideal if $Q$ is $G$-stable such that whenever $I, J$ are $G$-stable ideals of $A$ with $I J \subseteq Q$, either $I \subseteq Q$ or $J \subseteq Q$. A Poisson algebra $A$ is said to be $G$-simple if 0 and $A$ are the only $G$-stable Poisson ideals of $A$.

Lemma. For each admissible set $T,\langle T\rangle$ is an $H$-prime Poisson ideal of $A_{n}$.

Proof: Since every element of $T$ is $H$-eigenvector, $\langle T\rangle$ is $H$-stable and thus the result follows from 1.4.

2.3. Let an affine algebraic group $G$ act on a k-algebra $A$ by algebra automorphisms. Remind that the action $G$ on $A$ is said to be rational if $A$ is a direct union of finite dimensional $G$-invariant subspaces $V_{i}$ such that the restrictions $G \longrightarrow$ Aut $A \longrightarrow \mathrm{GL}\left(V_{i}\right)$ are morphisms of algebraic varieties.

Lemma . Every $H$-prime Poisson ideal of $A_{n}$ is a prime Poisson ideal.

Proof: Note that $H$ is an irreducible affine algebraic group since its coordinate ring is the prime ring

$$
\mathbf{k}\left[z_{1}^{ \pm 1}, z_{2}^{ \pm 1}, \ldots, z_{2 n}^{ \pm 1}\right] /\left\langle z_{1} z_{2}-z_{2 i-1} z_{2 i} \mid i=2, \ldots, n\right\rangle .
$$

Moreover $H$ acts rationally on $A_{n}$ by algebra automorphisms since $H$ acts semisimply with rational eigenvalues. Hence every $H$-prime Poisson ideal is a prime Poisson ideal by [1, II.2.9 Proposition]. (In the proof of [1, II.2.9 Proposition], replace the k-torus by an irreducible affine algebraic group.)

TheOREM 2.4. Every $H$-prime Poisson ideal of $A_{n}$ is generated by an admissible set.

Proof: Let $P$ be an $H$-prime Poisson ideal of $A_{n}$ such that $P \cap \mathcal{P}_{n}=T$. Then $T$ is an admissible set by 2.3 and 1.4. By way of contradiction, suppose that $P \neq\langle T\rangle$. Express each element of $\bar{A}_{n}=A_{n} /\langle T\rangle$ by a linear combination of elements of $\mathcal{B}_{T}$ given in 1.5 Lemma (b). Choose a nonzero element $f \in \bar{P}$ such that $f$ has the shortest length among those elements and let

$$
H_{i}=\left\{\left(h_{1}, h_{1}^{-1}, h_{2}, h_{2}^{-1}, \ldots, h_{n}, h_{n}^{-1}\right) \in H \mid h_{j}=1 \text { for all } j \neq i\right\} .
$$

Suppose that $y_{i} \in T$ and $x_{i} \notin T$. Applying $H_{i}$ on $f$, the degrees of all nonzero terms of $f$ with respect to $\bar{x}_{i}$ are equal since each element $z \in \mathcal{B}_{T}$ is an eigenvector of $H_{i}$ with 
eigenvalue depending on the degree of $z$ with respect to $\bar{x}_{i}$. Hence we may assume that the degree of $f$ with respect to $\bar{x}_{i}$ is zero since $x_{i} \notin P$ and $P$ is a prime ideal by 2.3 . By the same way the degree of $f$ with respect to $\bar{y}_{i}$ is zero, where $y_{i} \notin T$ and $x_{i} \in T$.

Suppose that $\Omega_{i} \in T, y_{i} \notin T, x_{i} \notin T$. For $z \in \mathcal{B}_{T}$, let $r$ and $s$ be the respective degrees of $z$ with respect to $\bar{y}_{i}$ and $\bar{x}_{i}$. Then $r s=0$ and, for each $h \in H_{i}, z$ is an eigenvector of $h$ with eigenvalue $c^{r-s}$, where $c$ is the $(2 i-1)$-th component of $h$. Hence, applying $H_{i}$ on $f$, the degrees of all nonzero terms of $f$ with respect to $\bar{y}_{i}$ (respectively, $\bar{x}_{i}$ ) are equal. Therefore we may assume that the degree of $f$ with respect to $\bar{y}_{i}$ (respectively, $\bar{x}_{i}$ ) is zero since $y_{i}, x_{i} \notin P$ and $P$ is a prime ideal by 2.3 .

Finally suppose that $\Omega_{i} \notin T$. For $z \in \mathcal{B}_{T}$, let $r$ and $s$ be the respective degrees of $z$ with respect to $\bar{y}_{i}$ and $\bar{x}_{i}$. Then $z$ is an eigenvector of $h \in H_{i}$ with eigenvalue $c^{r-s}$, where $c$ is the $(2 i-1)$-th component of $h$. Thus, applying $H_{i}$ on $f$, the differences between the degrees of all nonzero terms of $f$ with respect to $\bar{y}_{i}$ and those with respect to $\bar{x}_{i}$ are equal. Hence $f$ is the product of $\bar{y}_{i}^{r}$ ( or $\overrightarrow{x_{i}}$ ), $r \geqslant 0$, and a linear combination of elements $\bar{z}, z \in \mathcal{B}_{T}$, such that the degree of $z$ with respect to $\bar{y}_{i}$ is equal to that of $z$ with respect to $\bar{x}_{i}$. Since $y_{i}, x_{i} \notin P$ and $P$ is a prime ideal by 2.3 , we may assume that $f$ is a linear combination of elements $z \in \mathcal{B}_{T}$, such that the degree of $z$ with respect to $\bar{y}_{i}$ is equal to that of $z$ with respect to $\bar{x}_{i}$. Replace $\bar{y}_{j} \bar{x}_{j}$ in $f$ by $\left(q_{j}-p_{j}\right)^{-1} \bar{\Omega}_{j}-\bar{\Omega}_{j-1}$ for each $\Omega_{j} \notin T$ since $\left(q_{j}-p_{j}\right) y_{j} x_{j}=\Omega_{j}-\Omega_{j-1}$. Then we have that $f$ is a polynomial with variables $\bar{\Omega}_{j}$ such that $\Omega_{j} \notin T$.

That is, $P /\langle T\rangle$ contains a nonzero element which is a polynomial with variables $\bar{\Omega}_{j}$ such that $\Omega_{j} \notin T$. Note that $\bar{\Omega}_{j}$ 's, $\Omega_{j} \notin T$, are algebraically independent over $\mathbf{k}$ by 1.5 Lemma (c). Suppose that $g \in P /\langle T\rangle$ has the smallest length among such elements. Denote

$$
g=\sum_{i=1}^{m} a_{i} \bar{\Omega}_{j_{1}}^{r_{i 1}} \bar{\Omega}_{j_{2}}^{r_{i 2}} \cdots \bar{\Omega}_{j_{k}}^{r_{k}}
$$

where

$$
a_{i} \neq 0, \quad\left\{\Omega_{j_{1}}, \ldots, \Omega_{j_{k}}\right\}=\left\{\Omega_{i} \mid \Omega_{i} \notin T\right\}
$$

and

$$
j_{1}<j_{2}<\cdots<j_{k} .
$$

For each $x_{j}$ such that $\Omega_{j} \notin T$, there exists a derivation $\psi_{x_{j}}$ on the localisation $\left(A_{n} /\langle T\rangle\right)\left[\bar{x}_{j}^{-1}\right]$ defined by

$$
\psi_{x_{j}}(a)=\left\{\bar{x}_{j}, a\right\} \bar{x}_{j}^{-1}
$$

for all

$$
a \in\left(A_{n} /\langle T\rangle\right)\left[\bar{x}_{j}^{-1}\right] .
$$

Moreover the extension $\bar{P}^{e}$ is stable under $\psi_{x_{j}}$ and $g$ is an eigenvector of $\psi_{x_{j}}$ by (2). Now acting $H$ on $g$ and applying $\psi_{x_{j_{k}}}, \psi_{x_{j_{k-1}}}, \ldots, \psi_{x_{j_{2}}}$ to $g$, by (2), we have the following linear 
system

$$
\begin{aligned}
r_{i 1}+r_{i 2}+\cdots+r_{i k} & =c \\
\left(r_{i 1}+r_{i 2}+\cdots+r_{i, k-1}\right) p_{j_{k}}+r_{i k} q_{j_{k}} & =c_{k} \\
\left(r_{i 1}+r_{i 2}+\cdots+r_{i, k-2}\right) p_{j_{k}}+\left(r_{i, k-1}+r_{i k}\right) q_{j_{k-1}} & =c_{k-1} \\
\cdots & \\
r_{i 1} p_{j_{2}}+\left(r_{i 2}+r_{i 3}+\cdots+r_{i k}\right) q_{j_{2}} & =c_{2}
\end{aligned}
$$

for all $i=1,2, \ldots, m$, where $c, c_{k}, c_{k-1}, \ldots, c_{2}$ are constants which are independent to $i$. Since $p_{i} \neq q_{i}$ for all $i$, we have from (4) that $r_{1 \ell}=r_{2 \ell}=\cdots=r_{m \ell}$ for all $\ell=1,2, \ldots, k$ and thus $g$ is of the form $\alpha \bar{\Omega}_{j_{1}}^{1} \bar{\Omega}_{j_{2}}^{r_{2}} \cdots \bar{\Omega}_{j_{k}}^{r_{k}}$ for some nonzero $\alpha \in \mathbf{k}$. It follows that $P$ contains some $\Omega_{j}$ which is not in $T$, a contradiction.

2.5. For a Poisson algebra $A$, denote by $\operatorname{pspec}(A)$ the set of all prime Poisson ideals of $A$. For each ideal $I$ of $A_{n}$, denote by $(I: H)$ the largest $H$-stable ideal contained in $I$ and, for a $H$-prime Poisson ideal $J$ of $A_{n}$ and an admissible set $T$, set

$$
\begin{aligned}
& \operatorname{pspec}_{J}\left(A_{n}\right)=\left\{P \in \operatorname{pspec}\left(A_{n}\right) \mid(P: H)=J\right\} \\
& \operatorname{pspec}_{T}\left(A_{n}\right)=\left\{P \in \operatorname{pspec}\left(A_{n}\right) \mid P \cap \mathcal{P}_{n}=T\right\} .
\end{aligned}
$$

\section{Proposition.}

$$
\begin{aligned}
\operatorname{pspec}\left(A_{n}\right) & =\biguplus_{J H \text {-prime Poisson ideal }} \operatorname{pspec}_{J}\left(A_{n}\right) \\
& =\biguplus_{T \text { admissible set }} \operatorname{pspec}_{T}\left(A_{n}\right) .
\end{aligned}
$$

Proof: Let $P$ be a prime Poisson ideal of $A_{n}$. For $H$-stable ideals $I, J$, suppose that $I J \subseteq(P: H)$. Then $P$ contains $I$ or $J$ because $P$ is a prime ideal, hence $(P: H)$ contains $I$ or $J$ and thus $(P: H)$ is $H$-prime. Moreover $(P: H)$ is an $H$-prime Poisson ideal since $(P: H)=\bigcap_{h \in H} h(P)$ and every element $h \in H$ acts as a Poisson automorphism. Now the statement follows immediately from 2.4 and 1.4 .

\section{The group of Poisson aUtomorphisms of $A_{n, \Gamma}^{P, Q}$}

Lemma 3.1. Let $A$ be a finitely generated Poisson algebra, a any normal element which is not a unit, and $P$ a prime Poisson ideal minimal over $a A$. Then $P$ has height at most 1 .

Proof: If $Q$ is a prime ideal such that $a A \subseteq Q \subseteq P$ then the maximal Poisson ideal contained in $Q$ is a prime Poisson ideal containing $a A$ by $[3,3.3 .2]$, and thus $P=Q$. It follows that $P$ is a prime ideal minimal over $a A$. Hence $P$ has height at most 1 by $[7,4.1 .11]$. 
3.2. Let $\mathbf{N}$ be the set of all nonnegative integers. Now we give an order $\ll$ on the set $\mathbf{N}^{2 n}$ defined by

$$
\begin{gathered}
\left(r_{1}, r_{2}, \ldots, r_{2 n}\right) \\
\Longleftrightarrow\left(s_{1}, s_{2}, \ldots, s_{2 n}\right) \\
r_{2 n}=s_{2 n}, r_{2 n-1}=s_{2 n-1}, \ldots, r_{i+1}=s_{i+1}, \text { and } r_{i}<s_{i} .
\end{gathered}
$$

Every element of $A_{n}$ can be uniquely written as a linear combination of standard monomials $X^{\alpha}=y_{1}^{r_{1}} x_{1}^{r_{2}} y_{2}^{r_{3}} x_{2}^{r_{4}} \cdots y_{n}^{r_{2 n-1}} x_{n}^{r_{2 n}}$. For $0 \neq f \in A_{n}$ expressed by

$$
f=\sum_{\alpha \in \mathbb{N}^{2 n}} c_{\alpha} X^{\alpha}
$$

define $\exp (f)$ to be the maximal element in the set $\left\{\alpha \mid c_{\alpha} \neq 0\right\}$ under the order $\ll$. It is easy to see that $\exp (f g)=\exp (f)+\exp (g)$ for all nonzero elements $f, g \in A_{n}$.

Let $\mathbf{V}_{n}$ be the $\mathbf{k}$-vector space spanned by $\Omega_{1}, \Omega_{2}, \ldots, \Omega_{n}$. Note that $y_{1} x_{1}, y_{2} x_{2}, \ldots$, $y_{n} x_{n}$ form a $\mathbf{k}$-basis for $\mathbf{V}_{n}$ since $\Omega_{i}=\left(q_{i}-p_{i}\right) y_{i} x_{i}+\Omega_{i-1}$.

LEMMA 3.3. Suppose that the set of all prime Poisson ideals of $A_{n}$ with height 1 is

$$
\mathbf{P}=\left\{\left\langle y_{1}\right\rangle,\left\langle x_{1}\right\rangle,\left\langle\Omega_{2}\right\rangle,\left\langle\Omega_{3}\right\rangle, \ldots,\left\langle\Omega_{n}\right\rangle\right\}
$$

and let $q_{1} \neq 0$. If $\sigma$ is a Poisson automorphism of $A_{n}$ then for any $i \in\{2,3, \ldots, n\}$, there exists $j \in\{2,3, \ldots, n\}$ such that

$$
\sigma\left(\Omega_{i}\right)=\lambda_{i j} \Omega_{j}, \sigma\left(y_{1}\right)=r_{1} y_{1}, \sigma\left(x_{1}\right)=s_{1} x_{1}
$$

for some $\lambda_{i j}, r_{1}, s_{1} \in \mathbf{k}^{*}$.

Proof: For each $P \in \mathbf{P}, P$ is generated by a normal element $x$ which is not a unit. Since $0 \neq \sigma(P)$ is generated by a normal element $\sigma(x), \sigma(P)$ is a prime Poisson ideal with height 1 by 3.1 , and thus $\sigma(P) \in \mathbf{P}$. That is, $\mathbf{P}$ is invariant by $\sigma$. Let $x \in\left\{y_{1}, x_{1}, \Omega_{2}, \ldots, \Omega_{n}\right\}$. Then there exist $h, h^{\prime} \in A_{n} \backslash\{0\}$ and $y \in\left\{y_{1}, x_{1}, \Omega_{2}, \ldots, \Omega_{n}\right\}$ such that $\sigma(x)=h y$ and $\sigma^{-1}(y)=h^{\prime} x$. Hence $h \sigma\left(h^{\prime}\right)=\sigma\left(h^{\prime}\right) h=1$, and so $h, h^{\prime}$ are invertible. It follows that $h, h^{\prime} \in \mathbf{k}^{*}$.

Suppose now that there exist $i \in\{2,3, \ldots, n\}$ and $j \in\{2,3, \ldots, n\}$ such that $\sigma\left(\Omega_{i}\right)=\alpha y_{1}, \sigma\left(\Omega_{j}\right)=\beta x_{1}$ for some $\alpha, \beta \in \mathbf{k}^{*}$. Since $q_{1} \neq 0$ and $\left\{\Omega_{i}, \Omega_{j}\right\}=0$ by (2), applying $\sigma$ to this equality we get $\alpha \beta=0$. So for each $i \in\{2,3, \ldots, n\}$ there exists $j \in\{2,3, \ldots, n\}$ such that $\sigma\left(\Omega_{i}\right)=\lambda_{i j} \Omega_{j}$ for some $\lambda_{i j} \in \mathbf{k}^{*}$.

If $\sigma\left(y_{1}\right)=\alpha x_{1}, \sigma\left(x_{1}\right)=\beta y_{1}, \alpha, \beta \in \mathbf{k}^{*}$ then $2 q_{1} \alpha \beta=0$ by applying $\sigma$ to the equality $\left\{x_{1}, y_{1}\right\}=q_{1} x_{1} y_{1}$ given in (1). Therefore $\sigma\left(y_{1}\right)=r_{1} y_{1}, \sigma\left(x_{1}\right)=s_{1} x_{1}$ for some $r_{1}, s_{1} \in \mathbf{k}^{*}$.

LEMMA 3.4. Let $f, g \in A_{n} \backslash \mathbf{k}$ such that $f g=\sum_{1 \leqslant i \leqslant n} c_{i} y_{i} x_{i} \in \mathbf{V}_{n}$ with $c_{i} \in \mathbf{k}$ and $c_{n} \neq 0$. Then there exist $\lambda, \lambda^{\prime} \in \mathbf{k}^{*}$ such that $f=\lambda y_{n}$ and $g=\lambda^{\prime} x_{n} \quad$ (or $f=\lambda^{\prime} x_{n}$ and $\left.g=\lambda y_{n}\right)$. 
Proof: It is clear that $\exp (f g)=(0,0, \ldots, 1,1)$, so we have

$$
\exp (f)=(0,0, \ldots, 1,0), \quad \exp (g)=(0,0, \ldots, 0,1)
$$

or

$$
\exp (f)=(0,0, \ldots, 0,1), \quad \exp (g)=(0,0, \ldots, 1,0) .
$$

Then we have, for example,

$$
f=\lambda y_{n}+f_{0}, g=\lambda^{\prime} x_{n}+g_{0}
$$

for some $\lambda, \lambda^{\prime} \in \mathbf{k}^{*}, \quad f_{0} \in A_{n-1}$ and $g_{0} \in A_{n-1}\left[y_{n}\right]$. So

$$
f g=\lambda \lambda^{\prime} y_{n} x_{n}+\lambda^{\prime} f_{0} x_{n}+\lambda g_{0} y_{n}+f_{0} g_{0}
$$

and

$$
u=\lambda^{\prime} f_{0} x_{n}+\lambda g_{0} y_{n}+f_{0} g_{0} \in \mathbf{V}_{n}
$$

But

$$
\exp \left(\lambda^{\prime} f_{0} x_{n}\right)=\exp (u)=(\nu, 0,1)
$$

for some $\nu \in \mathbf{N}^{2(n-1)}$. It follows that $u \notin V_{n}$ if $f_{0} \neq 0$. Hence we have that $f_{0}=g_{0}=0$. By the same way we get the other case.

LEMMA 3.5. Suppose that the set of all prime Poisson ideals of $A_{n}$ with height 1 is

$$
\mathbf{P}=\left\{\left\langle y_{1}\right\rangle,\left\langle x_{1}\right\rangle,\left\langle\Omega_{2}\right\rangle,\left\langle\Omega_{3}\right\rangle, \ldots,\left\langle\Omega_{n}\right\rangle\right\} .
$$

Let $\sigma$ be a Poisson automorphism of $A_{n}$ and let $q_{i} \neq 0$ for all $i=1,2, \ldots, n$. Then for each $i \in\{1,2, \ldots n\}$, we have

$$
\sigma\left(\Omega_{i}\right)=\lambda_{i} \Omega_{i}, \sigma\left(y_{i}\right)=r_{i} y_{i}, \sigma\left(x_{i}\right)=s_{i} x_{i}
$$

for some $\lambda_{i}, r_{i}, s_{i} \in \mathbf{k}^{*}$.

PROOF: For $i=1$, we know that $\sigma\left(y_{1}\right)=r_{1} y_{1}, \sigma\left(x_{1}\right)=s_{1} x_{1}, r_{1}, s_{1} \in \mathbf{k}^{*}$ by 3.3 , and so $\sigma\left(\Omega_{1}\right)=\lambda_{1} \Omega_{1}$, where $\lambda_{1}=r_{1} s_{1}$. Suppose that there exist $i \neq j \in\{2,3, \ldots, n\}$ such that $\sigma\left(\Omega_{i}\right)=\lambda \Omega_{j}, \lambda \in \mathbf{k}^{*}$. Let $m$ be the maximal element in the set

$$
\left\{j \mid \sigma\left(\Omega_{l}\right)=\lambda \Omega_{j} \text { for some } \lambda \in \mathbf{k}^{*} \text { and } l \neq j\right\} \text {. }
$$

Let $\sigma\left(\Omega_{i}\right)=\lambda_{0} \Omega_{m}, \lambda_{0} \in \mathbf{k}^{*}$. Note that

$$
1<i<m \leqslant n, \sigma\left(\Omega_{i-1}\right)=\lambda^{\prime} \Omega_{r}, \sigma\left(\Omega_{i+1}\right)=\lambda^{\prime \prime} \Omega_{s}
$$

for some $\lambda^{\prime}, \lambda^{\prime \prime} \in \mathbf{k}^{*}$ and $r, s<m$. Applying $\sigma$ to $\Omega_{i}=\left(q_{i}-p_{i}\right) y_{i} x_{i}+\Omega_{i-1}$, we get

$$
\sigma\left(y_{i}\right) \sigma\left(x_{i}\right)=\sum_{1 \leqslant l \leqslant m} k_{l} y_{l} x_{l}
$$


with

$$
k_{m}=\left(q_{i}-p_{i}\right)^{-1}\left(q_{m}-p_{m}\right) \lambda_{0} .
$$

By 3.4 (with $m=n$ ) applied to $\sigma\left(y_{i}\right) \sigma\left(x_{i}\right)$, we have for example $\sigma\left(y_{i}\right)=\mu^{\prime} x_{m}, \mu^{\prime} \in \mathbf{k}^{*}$. If we apply $\sigma$ to $\Omega_{i+1}=\left(q_{i+1}-p_{i+1}\right) y_{i+1} x_{i+1}+\Omega_{i}$, then we get $\sigma\left(y_{i+1}\right)=\mu^{\prime \prime} x_{m}, \mu^{\prime \prime} \in \mathbf{k}^{*}$ (or $\left.\sigma\left(x_{i+1}\right)=\mu^{\prime \prime} x_{m}\right)$, which is a contradiction to the injectivity of $\sigma$. In conclusion we have $\sigma\left(\Omega_{i}\right)=\lambda_{i} \Omega_{i}$ for some $\lambda_{i} \in \mathbf{k}^{*}$. Now applying $\sigma$ to

$$
\Omega_{i}=\left(q_{i}-p_{i}\right) y_{i} x_{i}+\Omega_{i-1}, i=2,3, \ldots, n,
$$

we get $\sigma\left(y_{i}\right) \sigma\left(x_{i}\right) \in \mathrm{V}_{n}$. By $3.4(i=n)$ applied to $\sigma\left(y_{i}\right) \sigma\left(x_{i}\right)$, we have either $\sigma\left(y_{i}\right)$ $=r_{i} y_{i}, \sigma\left(x_{i}\right)=s_{i} x_{i}$ or $\sigma\left(x_{i}\right)=r_{i} y_{i}, \sigma\left(y_{i}\right)=s_{i} x_{i}$ for some $r_{i}, s_{i} \in \mathbf{k}^{*}$.

Finally, if $\sigma\left(x_{i}\right)=r_{i} y_{i}, \sigma\left(y_{i}\right)=s_{i} x_{i}$ then one have

$$
\begin{aligned}
\sigma\left(\left\{x_{i}, y_{i}\right\}\right) & =q_{i} r_{i} s_{i} y_{i} x_{i}+\lambda_{i-1} \Omega_{i-1} \\
\left\{\sigma\left(x_{i}\right), \sigma\left(y_{i}\right)\right\} & =-q_{i} r_{i} s_{i} y_{i} x_{i}-r_{i} s_{i} \Omega_{i-1}
\end{aligned}
$$

and thus we have $r_{i} s_{i}=0$, a contradiction. It completes the statement.

Theorem 3.6. Suppose that the set of all prime Poisson ideals of $A_{n}$ with height 1 is

$$
\mathbf{P}=\left\{\left\langle y_{1}\right\rangle,\left\langle x_{1}\right\rangle,\left\langle\Omega_{2}\right\rangle,\left\langle\Omega_{3}\right\rangle, \ldots,\left\langle\Omega_{n}\right\rangle\right\}
$$

and $q_{i} \neq 0$ for each $i=1,2, \ldots, n$. Then the group of Poisson automorphisms of $A_{n}$ is equal to the multiplicative group $H$.

Proof: Clearly $h=\left(h_{1}, h_{2}, \ldots, h_{2 n-1}, h_{2 n}\right) \in H$ is a Poisson automorphism. Conversely, if $\sigma$ is a Poisson automorphism of $A_{n}$ then $\sigma\left(y_{i}\right)=r_{i} y_{i}, \sigma\left(x_{i}\right)=s_{i} x_{i}$ for some $r_{i}, s_{i} \in \mathbf{k}^{*}$ by 3.5 . Since

$$
\begin{aligned}
& \sigma\left(\Omega_{n}\right)=\lambda_{n} \Omega_{n}=\sum_{1 \leqslant i \leqslant n}\left(q_{i}-p_{i}\right) \lambda_{n} y_{i} x_{i} \\
& \sigma\left(\Omega_{n}\right)=\sum_{1 \leqslant i \leqslant n}\left(q_{i}-p_{i}\right) \sigma\left(y_{i} x_{i}\right)=\sum_{1 \leqslant i \leqslant n}\left(q_{i}-p_{i}\right) r_{i} s_{i} y_{i} x_{i}
\end{aligned}
$$

we have that $r_{i} s_{i}=\lambda_{n}$ for each $i=1,2, \ldots, n$. It follows that $\sigma$ can be identified to $\left(r_{1}, s_{1}, \ldots, r_{n}, s_{n}\right) \in H$, as required.

3.7. Here we assume that

$$
q_{i}=-2, p_{i}=0, \gamma_{i j}=1
$$

for each $i$ and for each $j>i$. Then $A_{n}$ is called the coordinate ring of Poisson symplectic $2 n$-space.

Corollary. The group of Poisson automorphisms of the coordinate ring $A_{n}$ of Poisson symplectic $2 n$-space is the multiplicative group $H$.

Proof: By 3.6, it is enough to prove that the set of all prime Poisson ideals of $A_{n}$ with height 1 is

$$
\mathbf{P}=\left\{\left\langle y_{1}\right\rangle,\left\langle x_{1}\right\rangle,\left\langle\Omega_{2}\right\rangle,\left\langle\Omega_{3}\right\rangle, \ldots,\left\langle\Omega_{n}\right\rangle\right\}
$$


Clearly all elements of $\mathbf{P}$ are prime Poisson ideals of $A_{n}$ with height 1 by 2.5 . Let $P$ be a prime Poisson ideal with height 1 and suppose that $(P: H)=0$. Then $P$ does not contain any elements $y_{i}, \Omega_{i}, i=1, \ldots, n$ since they are $H$-eignevectors. Set

$$
B=A_{n}\left[y_{1}^{-1}, y_{2}^{-1}, \ldots, y_{n}^{-1}, \Omega_{1}^{-1}, \Omega_{2}^{-1}, \ldots, \Omega_{n}^{-1}\right] \text {. }
$$

By $[8$, Section 2] or $[10,2.2$ and 2.3], the algebra $B$ is presented by the Poisson algebra $\mathbf{k}_{u}\left(\mathbb{Z}^{2 n}\right)$, where $u: \mathbb{Z}^{2 n} \times \mathbb{Z}^{2 n} \longrightarrow \mathbf{k}$ is an antisymmetric biadditive map defined by the skew-symmetric $2 n \times 2 n$-matrix

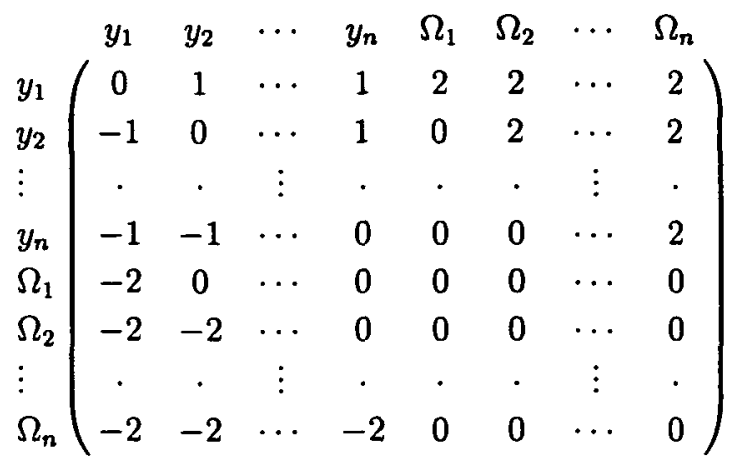

Since the determinant of the matrix (5) is nonzero, the radical of $u$

$$
\left\{\alpha \in \mathbb{Z}^{2 n} \mid u(\alpha, \beta)=0 \text { for all } \beta \in \mathbb{Z}^{2 n}\right\}
$$

is trivial, thus $\mathbf{k}_{u}\left(\mathbb{Z}^{2 n}\right)$ has no nonzero prime Poisson ideal by $[8,2.3]$. But the extension of $P$ to $B$ is a nonzero prime Poisson ideal, a contradiction. Hence $(P: H) \neq 0$. It follows that $P \in \mathrm{P}$ by 2.4 and 2.5 , as required.

\section{REFERENCES}

[1] K.A. Brown and K.R. Goodearl, Lectures on algebraic quantum groups, Advanced courses in mathematics - CRM Barcelona (Birkhäuser Verlag, Basel, Boston, Berlin, 2002).

[2] D. Cox, J. Little and D. O'Shea, Ideals, varieties and algorithms, Undergraduate Texts in Mathematics (Springer-Verlag, New York, 1997).

[3] J. Dixmier, Enveloping algebras, Graduate Studies in Mathematics 11, (American Mathematical Society, Providence, R.I., 1996).

[4] J. Gómez-Torrecillas and L. EL Kaoutit, 'Prime and primitive ideals of a class of iterated skew polynomial rings', J. Algebra 244 (2001), 186-216.

[5] J. Gómez-Torrecillas and L. EL Kaoutit, 'The group of automorphisms of the coordinate ring of quantum symplectic space', Beiträge Algebra Geom. 43 (2002), 597-601.

[6] K.L. Horton, 'The prime and primitive spectra of multiparameter quantum symplectic and Euclidean spaces', Comm. Algebra 31 (2003), 4713-4743.

[7] J.C. McConnell and J.C. Robson, Noncommutative Noetherian rings, Pure and Applied Mathematics (New York) (J. Wiley and sons, Chichester, 1987). 
[8] S-Q. Oh, 'Symplectic ideals of Poisson algebras and the Poisson structure associated to quantum matrices', Comm. Algebra 27 (1999), 2163-2180.

[9] S-Q. Oh, 'Poisson structures of multi-parameter symplectic and Euclidean spaces', (preprint).

[10] S-Q. Oh, C-G. Park and Y-Y. Shin, 'Quantum n-space and Poisson n-space', Comm. Algebra 30 (2002), 4197-4209.

Department of Mathematics

Chungnam National University

Daejeon 305-764

Korea

e-mail: sqoh@cnu.ac.kr
Department of Mathematics

Chungnam National University

Daejeon 305-764

Korea

e-mail: ehcho@math.cnu.ac.kr 